\title{
"Junté de mis papeles ese volumen": hacia una nueva edición de las Rimas sueltas de Juan de la Cueva ${ }^{1}$
}

\author{
Antonietta Molinaro \\ Università degli Studi di Napoli Federico II
}

Título: "Junté de mis papeles ese volumen": hacia una nueva edición de las Rimas sueltas de Juan de la Cueva.

Resumen: El presente trabajo constituye el primer acercamiento a una nueva edición crítica de la obra poética de Juan de la Cueva. Tras presentar la tradición textual del amplio corpus de rimas, constituida por un manuscrito autógrafo (el ms. 56-3-4 de la Biblioteca Colombina de Sevilla), una antología impresa por el autor (Obras, Sevilla, 1582) y algunos florilegios misceláneos de variada procedencia (entre los cuales destaca el mexicano Flores de baria poesía), se analizan los aspectos más peculiares y problemáticos con los que tiene que enfrentarse el editor de esta poesía. Por último, se proporciona un pequeño ejemplo de la investigación in fieri. En concreto, se considera un corpus de trece poemas, atestiguados por los tres testimonios fundamentales (Flores, Obras y autógrafo), que representan un muestrario privilegiado para rastrear algunos aspectos peculiares del proceso de escritura y revisión de Cueva a través de tres décadas de actividad poética.

Palabras clave: Juan de la Cueva, poesía áurea, rimas, filología de autor, manuscritos autógrafos.

Fecha de recepción: 22/3/2021.

Fecha de aceptación: 7/4/2021.
Title: "Junté de mis papeles ese volumen": Towards a New Edition of Juan de la Cueva's Rimas sueltas.

Abstract: This work is the first approach to a new critical edition of the poetic work of Juan de la Cueva. First of all, it describes the textual tradition of the extensive corpus of rhymes, consisting of an autograph manuscript (Sevilla, Biblioteca Colombina, ms. 56-3-4), an anthology printed during author's life (Obras, Sevilla, 1582) and some poetic miscellanies (among which the Mexican Flores de baria poesia stands out). Then, it analyses the most peculiar and problematic aspects that the editor of this poetry has to deal with. Finally, the paper focus on a group of thirteen poems, attested by the three fundamental testimonies (Flores, Obras and the autograph manuscript), which represent a privileged sample to trace some peculiar aspects of Cueva's writing and revision process through three decades of poetic activity, that will be further developed in the work of edition.

Key words: Juan de la Cueva, Golden Age poetry, Poetic Collection, Author's Philology, Autograph Manuscripts.

Date of Receipt: 22/3/2021.

Date of Approval: 7/4/2021.

1 Esta investigación se enmarca en el proyecto PRIN 2017, La tradizione del testo letterario in area iberica nel secolo d'oro, tra varianti d'autore e redazioni plurime (CUP E64I19002610006), dirigido por el profesor Antonio Gargano. 


\section{Un Volumen de Rimas a un paso de las PRENSAS}

Si muchos poetas del siglo XVI casi desdeñaban dar a la estampa sus obras, al pasar al nuevo siglo el escenario evoluciona lenta y constantemente hacia una conversión a letras de molde de sus versos de toda una vida. Pioneros de este cambio de rumbo son los autores sevillanos de la segunda mitad del Quinientos, que disfrutan del excepcional florecimiento económico y cultural que vivió la ciudad hispalense durante esos años ${ }^{2}$.

Sin embargo, aunque disfrutase de un contexto sociocultural de progresiva expansión de la poesía impresa y fuese más que consciente del inmenso potencial de los tipos móviles, Juan de la Cueva (Sevilla, 1543-Granada, 1612) no consiguió imprimir en vida una colección completa de sus versos. Dicha aserción se carga, además, de una nota de amargura, ya que este prolífico autor se destaca de los demás por un afán prolongado e incansable, en las últimas décadas de su vida, de recolección, revisión y, en algunos casos, incluso refundición de sus composiciones poéticas con el fin de transmitirlas, en su conjunto, a la posteridad. Su ambicioso proyecto editorial, que contaba con el amparo de su hermano Claudio de la Cueva - a quien iba dedicado-, preveía, junto a un volumen de poemas breves, rotulado De las Rimas de Juan de la Cueva, primera parte, un segundo, la Segunda parte de las Obras de Juan de la Cueva, con siete églogas y algunos poemas de mayor extensión ${ }^{3}$. A pesar de tantos esfuerzos, dichos gruesos

2 Ignacio García Aguilar, Poesía y edición en el Siglo de Oro, Madrid, Calambur, 2009, pp. 289-290.

3 Prescindiendo del contenido del primer volumen, sobre el que tendremos ocasión de volver en las páginas siguientes, solo indicamos aquí las obras contenidas en el segundo: églogas I-VII, Amores de Marte y Venus, Llanto de Venus, Historia de la Cueva, Viaje de Sannio, Ejemplar poético, De los inventores de las cosas, La Muracinda, Batalla entre ranas y ratones y, sin que conste en la tabla final, la epístola Olla guisada al sol, dijo un sofista, dirigida a Sayas de Alfaro. Algunas de estas obras mayores fueron copiadas nuevamente en limpio por Cueva, dando vida a otros autógrafos parciales. Al respecto, resultan útiles para una reconstrucción de la tradición textual del conjunto las ediciones críticas de José Cebrián García: Viaje de Sannio (Madrid, Miraguano, 1990), Églogas completas (Madrid, Miraguano, 1988), Fábulas mitológicas y épica burlesca (Madrid, Editora Nacional, 1984) y la de José María Reyes Cano, antepuesta a su edición del Exemplar poético (Sevilla, Alfar, 1986). 
códices autógrafos, que debían garantizar la divulgación masiva de las que el poeta había identificado en el prólogo general al primer volumen, respectivamente, como "rimas sueltas" y "obras continuadas" ${ }^{4}$, se quedaron a un paso de las prensas. Muerto su destinatario y estando Cueva postrado en la cama, los dos volúmenes fueron confiados, según las mandas testamentarias del autor, a la custodia de los frailes del convento de la Cartuja de Santa María de las Cuevas 5 . De allí pasaron luego a la Biblioteca Capitular y Colombina de Sevilla, donde actualmente se conservan bajo las signaturas: 56-3-4 (olim 82-2-4) y 56-3-5 (olim 82-2-5) -C1 y C2, según suelen ser identificados-.

El nombre de Juan de la Cueva sigue siendo hoy renombrado; sobre todo gracias a su pluma dramática y a las soluciones estéticas innovadoras que experimentó en sus piezas teatrales, por las que se le reconoce un papel destacado en el recorrido hacia la comedia nueva ${ }^{6}$. Sin embargo, el cuidado personal que el autor reservó, dentro de su amplia y heterogénea producción, a revisar y transmitir de sus composiciones de cuño italianista revela a las claras la importancia que él mismo les reconocía en su anhelo de fama y aspiración a un final asentamiento en el parnaso hispalense ${ }^{7}$.

4 De las Rimas de Juan de la Cueva, primera parte, f. 2v. Para no sobrecargar las notas, la referencia de todas las citas siguientes extraídas desde este manuscrito - a partir de ahora, C1 - se colocarán en el cuerpo del texto. La grafía se moderniza según las normas actuales. También se modernizan la puntuación, la acentuación y el uso de las mayúsculas. Se mantienen, en cambio, las contracciones y el apóstrofo, por su relevancia en la identificación de las sinalefas en los textos poéticos. La numeración a la que se acude es la que está escrita en lápiz en el manuscrito, la más coherente de las dos presentes, aunque pertenezca verosímilmente a una mano diferente de la del autor.

5 El testamento de Cueva puede leerse en José María Reyes Cano, "Documentos relativos a Juan de la Cueva: nuevos datos para su biografía”, Archivo Hispalense, LXIV, 196 (1981), pp. 107-135 (pp. 115-119).

6 Sobre el teatro de Cueva véase Francisco Javier Burguillo López, Juan de la Cueva y el nacimiento del teatro histórico en España, Salamanca, Universidad de Salamanca [tesis doctoral], 2010, al que remitimos también para una breve reconstrucción de los altibajos de la valoración crítica moderna del autor como dramaturgo a lo largo del siglo XX, sobre todo con relación al desarrollo de la comedia nueva.

7 Los estudios que abordan el tema, central para Cueva, de sus difíciles relaciones con sus pares —entre fricciones y críticas - en los círculos letrados y académicos sevillanos y de su consciencia con relación a una posición de exclusión $-\mathrm{y}$ au- 
Aun así, a diferencia de los poemas mayores, que finalmente llegaron, en tiempos más o menos recientes, a los tipos de la imprenta ${ }^{8}$, el rico corpus de rimas permanece inédito y de difícil acceso para los estudiosos, que siguen recurriendo al autógrafo para sus pesquisas e investigaciones. Además, y por si esta retahíla de adversidades fuera poco, el infortunio se repitió, al quedar sin publicar el trabajo de tesis doctoral de José María Reyes Cano, que representa el único intento de sacar de un olvido casi total la poesía de este escritor ${ }^{9}$. Con todo, algunos años más tarde vieron la luz un par de estudios filológicos de José Cebrián, cuyo alcance, sin embargo, resulta mucho más limitado por ceñirse a un reducido número

toexclusión- son varios. Entre ellos, véanse: Ignacio García Aguilar, "Juan de la Cueva: entre academia e imprenta”, Studi Ispanici, 43 (2018), pp. 123-153; las contribuciones de Francisco Javier Escobar Borrego, entre sí complementarias, "La obra poética de Juan de la Cueva en el entorno sevillano (con un excurso sobre sus vínculos con Diego Girón y Fernando de Herrera)", Rivista di Filologia e Letterature Ispaniche, 12 (2009), pp. 35-70; y "Juan de la Cueva, artifex exclusus: un poeta en los márgenes del Parnaso sevillano, a propósito del Viage de Sannio", en Compostella Aurea. Actas del VII Congreso de la AISO, eds. Antonio Azaustre Galiana, Santiago Fernández Mosquera, Santiago de Compostela, Universidad de Santiago de Compostela, 2008, pp. 263-270; Valentín Núñez Rivera, " $Y$ vivo solo y casi en un destierro: Juan de la Cueva en sus epístolas poéticas", en La epistola, ed. Begoña López Bueno, Sevilla, Universidad de Sevilla, 2000, pp. 257-294, que trata el asunto ciñéndose, en particular, en el amplio corpus de epístolas de Cueva.

8 Cfr. supra, nota 2.

9 José María Reyes Cano (ed.), Las Rimas de Juan de la Cueva, Barcelona, Universidad de Barcelona [tesis doctoral], 1982, 3 vols. Precisamos que, desde hace algunos meses, es posible consultar en libre acceso, a través del repositorio digital institucional de la Universidad de Barcelona, una versión digitalizada de la tesis mecanográfica original. Aprovechamos la ocasión para agradecer por su amabilidad y disponibilidad al profesor Rogelio Reyes Cano, que nos dio licencia para acceder al trabajo de su hermano antes de que dicha reproducción digital se culminase y estuviese disponible en línea. En cuanto al contenido de la tesis, pasando por alto la distinción entre los dos volúmenes autógrafos, Reyes Cano edita el contenido completo del manuscrito $\mathrm{C} 1$ y una parte de las obras copiadas en C2 (en concreto, las siete églogas, El llanto de Marte y Venus y Los amores de Marte y Venus) por considerarlas fruto de la misma inspiración lírica que las rimas sueltas. Por último, cabe recordar que dicha edición llegaba después de que el estudioso ya hubiera dedicado a la poesía de Cueva dos trabajos anteriores: "Juan de la Cueva, poeta lírico: un aspecto prácticamente inédito", Archivo Hispalense, LXI, 186 (1978), pp. 119-28; y La poesía lírica de Juan de la Cueva, Sevilla, Diputación de Sevilla, 1980. 
de poemas ${ }^{10}$. A pesar de todo, estas contribuciones tienen el gran mérito de haber puesto de relieve la riqueza y complejidad variantística de la producción poética cueviana y de haber abierto así el camino a unos estudios más extensos y completos.

Desde esta perspectiva, las páginas que siguen dan cuenta de las primeras calas de un trabajo in fieri que consiste precisamente en realizar una edición de este apreciable conjunto de rimas. Una edición que tendrá su punto de partida en la colección del autógrafo pero que, al mismo tiempo, ensanchará la mirada hacia una tradición textual algo más amplia, hecha por testimonios de variada procedencia. Se tratará así de definir con más precisión la personalidad poética de Cueva, sea con respecto a su íntimo proceso de creación, revisión y corrección textual, sea, complementariamente, en relación con su proyección en el panorama literario sevillano de esos años, para por fin llevar a buen término el proyecto personal del autor de dar a conocer su obra poética completa a través de la imprenta.

\section{MÁs ALLÁ DEL AUTÓGRAFo. La TRADICIÓN TEXTUAL DE UNAS RIMAS SUELTAS}

No hay duda de que el caso de Juan de la Cueva, con su colección de poemas autógrafos, representa una rareza en el panorama áureo y una fuente riquísima para el filólogo que decida emprender la tarea de su edición. Además, el códice de las Rimas de la biblioteca Colombina (C1) no solo es un testimonio extremadamente valioso de la última voluntad, tanto textual como estructural, del autor en cuanto a su producción poética suelta, sino que también constituye una traza concreta del largo y laborioso proceso que culminó, finalmente, en tan frustrado resultado.

10 Entre los muchos trabajos filológicos y críticos de Cebrián sobre la obra poética de nuestro autor, encontramos la edición de las composiciones de Cueva copiadas en las Flores de Baria Poesia ("Cueva en Flores de baria poesía", En la Edad de Oro. Estudios de ecdótica y crítica literaria, México, El Colegio de México, 1999, pp. 179220), y la de los poemas variamente relacionados con su experiencia biográfica en Nueva España (Juan de la Cueva y Nueva España. "Tú encendiste en amor el alma mía", Kassel, Reichenberger, 2001). 
Es el propio autor quien da cuenta de la naturaleza facticia de ese códice. En la dedicatoria a su hermano, al presentar el libro, además de resaltar la centralidad del gusto de los lectores a la hora de planificar su obra, Cueva proporciona algunas noticias, breves pero esenciales, sobre el proceso de confección:

Junté de mis papeles ese volumen hecho en diferentes tiempos, a varios propósitos, sin el principal que es amatorio. Hice división dél en dos partes: en la primera puse todas las rimas sueltas, mezclando con la variedad de sujetos las composiciones amatorias, misivas y burlescas, por variar los gustos a los lectores; en la segunda van las obras continuadas, en que no entiendo que se hallará menos gusto que en la variedad de las primeras $(\mathrm{C} 1, \mathrm{f} .2 \mathrm{v})$.

El códice $\mathrm{C} 1$, un grueso manuscrito en cuarto de 374 folios, lleva en la dedicatoria una fecha muy precisa: "1 de enero de 1603 " (f. $3 v)^{11}$. Sin embargo, esta ha de considerarse una datación orientativa, que probablemente señala el momento en que Cueva concibió el primer plan de la recopilación. De todas formas, la fecha citada no puede ser considerada la de concreción completa del conjunto, ya que en el manuscrito se acogen composiciones fechadas o fechables en años sucesivos, hasta, por lo menos, los años en los que el autor estuvo en Cuenca ${ }^{12}$. Al mismo tiempo, los replanteamientos tanto de la foliación como de la numeración progresiva de las composiciones, las distintas calidades del papel y la irregularidad del útil de escritura parecen reflejar una encuadernación en distintos momentos, con materiales de procedencia diferente.

Tras algunos textos preliminares (ff. $1 \mathrm{r}-15 \mathrm{v}$ ), en prosa y en verso, en parte de Cueva y en parte ajenos, que — como veremos a continuaciónrecuperan en buena medida los de un proyecto anterior, se inaugura la recopilación de los "Sonetos, canciones, elegías etc. de Juan de la Cueva" (f. 16r), constituida por 265 sonetos, 21 canciones, 23 elegías, 20 epístolas y

11 La portada también llevaba originariamente fecha, hoy ilegible debido a un corte del papel en ese punto.

12 José Cebrián García, "Para la biografía de Juan de la Cueva", en Estudios sobre Juan de la Cueva. "No tengo duda qu'estrañéis mi nombre", Sevilla, Universidad de Sevilla, 1991, pp. 15-66 (pp. 38 y ss.). 
1 sextina ${ }^{13}$, dispuestos según un criterio de variatio formal y temática. El códice resulta mútilo en la parte final, ya que se anuncia una epístola de encargo — verosímilmente la composición de despedida - de la que solo queda la rúbrica: "Epístola 19 al doctor Claudio de la Cueva, inquisidor apostólico, etc., en que le encarga que favorezca y ampare este libro que se le dedica y lo defienda, como protector dél, contra los ofensores de estas cosas" (f. 370v). Asimismo, se presenta inacabada la tabla final, que se interrumpe bruscamente en la letra $\mathrm{D}$. La presencia de un paratexto muy articulado (prólogo, dedicatoria, textos de homenaje de diferentes autores, composiciones del propio Cueva), la grafía discretamente cuidadosa, el método de corrección por banderillas y la existencia de varias enmiendas caligráficas, así como la uniformidad y regularidad de los titulillos ("Primera" en el verso y "Parte" en el recto que le sigue) y de los reclamos, dejan muy claro el estatuto final de copia en limpio de este códice, concebido verosímilmente para actuar como original de imprenta.

Acompañan a este testimonio, por así decirlo, definitivo, otros dos, ya conocidos por los críticos y esenciales para la reconstrucción de la obra poética de nuestro autor: el florilegio mexicano Flores de Baria Poesía (1577) y la antología impresa de las Obras (Sevilla, 1582).

El primero de los dos, Flores de Baria Poesía (F), es un manuscrito poético de 400 folios, estructurado en cinco libros ordenados temáticamente, del que se conservan hoy solo el primero y parte del segundo. Custodiado en la actualidad en la Biblioteca Nacional de Madrid (ms. 2973), es sobradamente conocido por su relevancia cultural en el panorama áureo al ser el primer cancionero colectivo de marco petrarquista del Nuevo Mundo ${ }^{14}$. Nuestro poeta, que vivió algunos años en México con su hermano Claudio durante su treintena (1574-1577), es efectivamente un componente esencial de este producto compartido, expresión de

13 Para ser más precisos, las epístolas son 19 ya que la que efectivamente debería de ser la $20-y$ que lleva el número 19 en la recopilación debido a la presencia de una epístola no numerada-, como se verá enseguida, no consta en el manuscrito, excepto por su epígrafe.

14 Cfr. Flores de Baria Poesía. Cancionero novohispano del siglo XVI, ed. Margarita Peña, México, FCE, 2004 [UNAM, 1980]. En tiempos más recientes, a esta edición se añadió la tesis doctoral (inédita) de María José Rodríguez Mosquera, Flores de Baria Poesía (México, 1577). Estudio y análisis del manuscrito, Barcelona, Universidad de Barcelona, 2013. 
unas tertulias literarias activas en los ambientes cultos de la colonia. De hecho, por entre las páginas del florilegio, entre poemas de autores —en su mayoría, sevillanos y novohispanos- y otros anónimos, destacan 32 composiciones rotuladas a nombre de Juan de la Cueva que le convierten en el segundo poeta más representado, después de Gutierre de Cetina ${ }^{15}$. De ahí que haya surgido la hipótesis, luego descartada con argumentaciones bastante concretas, de su papel como colector de la miscelánea ${ }^{16}$. Con respecto a sus composiciones ( 25 sonetos, 3 madrigales, 2 odas, 1 elegía y 1 sextina), solo 24 encontraron acogida en el volumen de Rimas sueltas mientras que de las 8 restantes, excluyendo 1 madrigal, que fue publicado en las Obras, 7 solo se trasmiten a través de este testimonio mexicano.

En cuanto al segundo testimonio antes mencionado, es decir, las Obras - a partir de ahora, $\mathrm{O}$ - nos permite averiguar que, aunque no pudo ver realizado el propósito final de publicar su obra poética completa, Juan de la Cueva logró participar activamente en el floreciente escenario editorial sevillano del último tercio del siglo $\mathrm{XVI}^{17}$. De hecho, en el año 1582, salió del taller de Andrea Pescioni, enmarcada en el proyecto de una "publicación continuada" 18 de antologías de diferentes autores hispalenses, una selección de sus composiciones de carácter amoroso, rotulada: Obras de Juan de la

15 Peña (ed.), op. cit., p. 41.

16 Sobre el asunto véanse, en particular, Peña (ed.), op. cit., pp. 27-33 y Cebrián, "Cueva en Flores de Baria Poesía", pp. 180-183. Sin embargo, hay que precisar que, aunque Cebrián haya descartado, tras un examen de tipo gráfico-fonético, la hipótesis de la composición de primera mano por parte de Cueva, sus conclusiones no rechazan la suposición de Peña relativa a una responsabilidad proyectual, aunque no materialmente ejecutoria, del códice por mano de este autor.

17 Ya se ha mencionado la condición de excepcionalidad en cuanto a la difusión de obras poéticas impresas en la ciudad hispalense en el Siglo de Oro. En cuanto a Cueva, pasando por alto lo que pasó con su obra poética de inspiración lírica — de la que se hablará a continuación-, el autor dio a la imprenta varias otras obras de diferentes géneros, entre las cuales destacan: una colección de obras teatrales estrenadas en Sevilla, la Primera parte de las comedias y tragedias (1583), una recopilación de romances históricos, el Coro febeo de romances historiales (1588), y un poema épico, La conquista de la Bética (1603).

18 Valentín Núñez Rivera, "1582 (Poesía, imprenta y canon)”, en El canon poético en el siglo XVI, ed. Begoña López Bueno, Sevilla, Universidad de Sevilla, 2008, pp. 141 175 (p. 142). 
Cueva $^{19}$. El tema amoroso actúa como un hilo conductor de las tres secciones de esta colección en cuarto de 139 folios, comenzando por una primera recopilación de sonetos, canciones, odas, elegías, sextinas y madrigales, todos de ascendencia petrarquista y dedicadas a la mujer amada, una tal Felipa de la Paz (ff. 13r-94r), continuando con una segunda sección bucólica, compuesta por tres églogas (ff. 95r-120r), para concluir, finalmente, con una fábula mitológica en octavas sobre el infeliz éxito de los amores de Venus y Adonis (ff. 121r-135v). Dejando a un lado las églogas y el Llanto de Venus que, en el proyecto sucesivo en dos volúmenes, cayeron entre las Obras continuadas, las 133 composiciones de la primera parte confluyeron, con la única excepción de 2 madrigales y 1 elegía, en las Rimas ${ }^{20}$. En definitiva, considerando también las compartidas con F, las Obras proporcionan 2 composiciones de testimonio único. De esta edición han llegado a nosotros 9 ejemplares, hoy conservados en diferentes bibliotecas europeas ${ }^{21}$.

Por tanto, las Flores y las Obras son igualmente testimonios fundamentales, no solo por incrementar el número de composiciones poéticas de 333 a 343, sino también por proporcionar redacciones anteriores de un

19 Junto con las Obras de Cueva, salen de ese taller otras tres antologías poéticas individuales: Algunas obras de Herrera, Obras de Cepeda y Églogas pastoriles de Padilla. Para las características de cada poemario, las semejanzas entre ellos y el significado de esta empresa editorial conjunta véanse García Aguilar, "Poesía y edición en el Siglo de Oro", pp. 225-228 y 293-298; y Núnez Rivera, "1582 (Poesía, imprenta y canon)", quien además destaca los elementos de proximidad entre Cueva y Herrera dentro de un contexto general de correferencias entre los poemarios impresos entre la segunda mitad del siglo XVI y la primera del XVII.

20 A la cifra de 131 hay que añadir las 3 composiciones de Cueva que se encuentran entre los textos preliminares (la elegía De cuántos has de ser reprehendido, la canción Con los despojos del Citereo asalto y el soneto Según te agrada, a cada uno aplicas) y que confluyen todas en las Rimas.

21 Reyes Cano detectó 5 ejemplares: 3 en la Biblioteca Nacional de Madrid (R/1566; R/3267; R/13333), 1 en la Biblioteca del Escorial (21.V.19(2)) y otro en la British Library de Londres (C.57.K.17). Una consulta del catálogo digital USTC (Universal Short Title Catalogue) nos permitió añadir otros 4, que se conservan en las bibliotecas de Chicago (Newberry Library, Y.722.C.907), Oxford (Codrington Library, nn.17.1), Salamanca (Biblioteca Universitaria, BG/11436) y Viena (Österreichische Nationalbibliothek, *38.Bb.39.PS). Para menciones futuras de dichos ejemplares se utilizarán, según la orden de este listado, las siguientes abreviaciones: $m_{1566} ; m_{3267}$; $m_{13333}$; esc; lon; chi; ox; sal; vie. 
buen número de poemas que tomaron su facies definitiva en las Rimas.

Aun así, la tradición textual de las composiciones de Cueva que podemos rotular como rimas es algo más extensa. Ante todo, a los textos mencionados, hay que añadir las 2 composiciones que el propio Cueva introdujo entre los preliminares de la Conquista de la Bética, en concreto, un soneto, Vivo esplendor de aquella inmortal llama, y una canción, Nuevo espiritu pide mi deseo, que no fueron incluidas posteriormente en el autógrafo. Pero, hay más. De hecho, aunque ya se haya insistido en la adhesión parcial y conflictiva de nuestro poeta a los círculos literarios coevos de su ciudad y, por consiguiente, su escasa representación en las manifestaciones poéticas colectivas, algunos estudios recientes y un detenido - aunque siempre perfectible- escrutinio de catálogos y otros repertorios bibliográficos han sacado a la luz unos cuantos testimonios adicionales de algunas composiciones recogidas en las Rimas, que tendrán que ser analizados con la debida atención en una nueva edición. Entre ellos, encontramos el ms. Span. 56 de la Houghton Library (Universidad de Harvard), que da acogida a 2 composiciones de Cueva: el soneto El que al rebelde apóstata detuvo, escrito con ocasión de la muerte del rey Felipe II, y la elegía fúnebre para Bárbara de la Cueva, Mueve espiritu sacro de Cyrrheo ${ }^{22}$. Al haber sido confeccionado por el intelectual y pintor Francisco Pacheco, amigo de Cueva y animador de unas tertulias académicas en Sevilla en las que muy probablemente participó nuestro autor ${ }^{23}$, este manuscrito se sitúa en

22 José Cebrián García, "Juan de la Cueva en el Cancionero Ms. Span 56 de la Houghton Library”, en De palabras, imágenes y símbolos. Homenaje a José Pascual Buxó, ed. Enrique Ballón Aguirre, México, UNAM, 2002, pp. 137-151. Para mayores detalles sobre el florilegio, su contenido, historia y otros aspectos de interés remitimos al reciente estudio de Juan Montero, "El taller poético del pintor Pacheco: el ms. Span 56 de la Houghton Library (Universidad de Harvard)", en Cancioneros del Siglo de Oro. Forma y formas, ed. Andrea Baldissera, Pavia, Ibis, 2019, pp. 167-181, y a la bibliografía allí mencionada.

23 Cfr. García Aguilar, "Juan de la Cueva: entre academia e imprenta", pp. 133-135. Sobre la existencia de una academia de Pacheco la crítica sigue, en realidad, bastante dividida. Véase, por ejemplo, Marta P. Cacho Casal, Francisco Pacheco y su "Libro de retratos", Sevilla/Madrid, Fundación Focus-Abengoa/Marcial Pons, 2011, quien -especialmente en las pp. 103-113- recupera y argumenta la idea, ya defendida por Bassegoda, de la existencia de un círculo de amigos que se reunía en la casa del pintor más que de una verdadera academia. 
una posición relevante en cuanto a la procedencia de sus textos y, por consiguiente, al estatuto de las variantes. En particular, transmite la única versión completa hoy conocida de la elegía, ya que resulta lagunosa la copia que de ella insertó Cueva en $\mathrm{C}^{24}$. Por otra parte, el propio Pacheco eligió este mismo soneto y otro, Concedeos la cumbre del Parnaso, también de nuestro autor, para acompañar los retratos, respectivamente, de Cristóbal de Sayas y Alfaro y del rey Felipe II en su Libro de descripción de verdaderos retratos (1599). Igualmente relacionados con el espacio social y académico hispalense son los testimonios de obras ajenas que incluyen en el paratexto literario composiciones encomiásticas de Cueva, aunque, por ahora, solo se haya detectado un caso, es decir, el del Verdadero entretenimiento del Christiano (1584) de Andrés de Losa, que abre su sección de composiciones de alabanza a la obra con la canción cueviana Si tu divina mano.

Por último, otros testimonios más son los que revelan también una precoz confusión de atribuciones. Es lo que pasa con relación a algunas composiciones de Cueva asignadas al ingenio de Barahona de Soto. A ese respecto, aunque se tenga que renunciar al ms. 33-180-6 de la Biblioteca Colombina de Sevilla, citado por todos los estudiosos de Cueva y de Barahona y hoy lamentablemente desaparecido, habrá que analizar el florilegio presuntamente vinculado a este, es decir, el ms. B-2369-91 de la Hispanic Society of America, y, además, el ms. 3708 de la Biblioteca Nacional de Madrid, ya que ambos trasmiten diversas composiciones de Cueva atribuyéndolas al poeta cantor de la Angélica ${ }^{25}$. Al lado de estos, mencionamos otros 2 florilegios de poesías diversas de la Biblioteca Nacional: el ms. MN 4117, que copia, entre algunos poemas de Góngora, el soneto cueviano Elena un día se miró al espejo, y el MN 10293, en el que se le atribuye a Baltasar del Alcázar la epístola Junto a la calle que dejando el nombre. Por último, desde una perspectiva complementaria a la de los

24 Fue el propio Cueva quien mutiló el texto al cortar parte del fascículo para reutilizar el grabado impreso en su portada en otra obra suya. Véanse, al respecto, las observaciones de Cebrián, "Juan de la Cueva en el Cancionero Ms. Span 56”, p. 141.

25 Sobre este asunto nos limitamos por ahora a remitir a la lectura cruzada de las dos entradas sobre "Luis Barahona de Soto" (por José Lara Garrido) y "Juan de la Cueva" (por José María Reyes Cano) del Diccionario filológico de literatura española, eds. Pablo Jauralde Pou, Delia Gavela García, Pedro C. Rojo Alique, Madrid, Castalia, 2009; respectivamente, pp. 108-120 y 334-342. En particular, es Lara Garrido quien relaciona el ms. americano con el códice perdido (ibidem, p. 111, nota 2). 
casos sobredichos, tampoco se pueden pasar por alto a priori esas composiciones que, aun no estando incluidas en ninguno de los testimonios principales, resultan asignadas a nuestro poeta por copistas anónimos en testimonios esporádicos, como sucede con el exitoso soneto No eres nieve, que fueras derretida, que sería de Cueva según el ms. MN 2856 y, asimismo, con las seis composiciones que el antólogo del cartapacio sevillano Poesias Varias De Diversos Authores em Castelhano de la Nacional de Lisboa (F.G. 3072) parece atribuir, todas o en parte, a Cueva ${ }^{26}$.

\section{Prolegómenos para una nueva edición: Problemas y MÉtodos De UNA TRADICIÓN MIXTA}

Como adelantamos, la única tentativa de realizar una edición crítica completa de las rimas de Juan de la Cueva permanece sin publicar. Con su extenso estudio, Reyes Cano aspiraba a sanar una prolongada ausencia en el campo de los estudios poéticos áureos, presentando una edición que enseńase las múltiples caras temáticas y formales de la inspiración poética de este marginado autor hispalense. Además de esto, por un lado, el estudio inicial y los aparatos llamaban la atención sobre "la labor de continua poda a la que sometió [Cueva] sus textos a lo largo de su vida" ${ }^{27}$ y, por el otro, sirviéndose sobre todo de los datos proporcionados por Bartolomé José Gallardo, el estudioso reconstruía con bastante detenimiento la escasa recepción del poeta sevillano (ss. XVII-XIX) y los problemas de autoría conectados con algunas de sus composiciones ${ }^{28}$. De todas formas, los resultados de su tesis nunca lograron estructurarse de una forma definitiva, para luego poder ser analizados críticamente ad locum. De hecho, los aparatos

26 En concreto, en el epígrafe a la composición núm. 64 del cartapacio se lee "Obras de Juan de las Cuevas". Debido a que la sucesiva noticia relativa a una atribución, diferente de esta, se encuentra en la rúbrica del poema núm. 70 , se ha planteado la hipótesis de que no solo el primero sino también los cinco textos de en medio fuesen atribuidos todos, por el anónimo antólogo, a Juan de la Cueva. Cfr. Juan Montero, "Dos textos poéticos de Fernando de Herrera con variantes y un posible soneto desconocido (más una lira antiherreriana)", Bulletin Hispanique, CVII, 2 (2005), pp. 605-616 (pp. 614-616).

27 Reyes Cano (ed.), “Las Rimas de Juan de la Cueva”, p. X.

28 Ibidem, pp. XV-XVI y XIX-XXV. 
establecidos se configuran en una estructura poco articulada, que prevé una colocación promiscua de las lecciones de los testimonios relacionados con el autor, como lo son las Flores y las Obras, justo al lado de ediciones poco o nada científicas de los siglos XVIII-XX, sin que se pueda distinguir de inmediato entre variantes de autor y de transmisión ${ }^{29}$. Además, el análisis crítico de estos datos filológicos ha permanecido, por así decirlo, en una fase embrionaria de desarrollo, con una presentación de la varia lectio observada que, sin embargo, no ha recibido una atención específica a lo largo del estudio introductorio ni en otra ocasión sucesiva. Además, desde la realización de la investigación de Reyes Cano se han editado catálogos y repertorios que, como se ha mostrado en el apartado anterior, abren al estudioso de hoy un universo de nuevos fondos y colecciones, permitiendo una potencial extensión de los testimonios, con lecciones distintas de los poemas conocidos e, incluso, posibles nuevas atribuciones ${ }^{30}$.

Por su parte, en sus dos ediciones parciales, Cebrián solo considera los tres testimonios fundamentales ${ }^{31}$ y nunca se abre a comentarios, ni con relación a la cronología de las versiones por ellos proporcionadas, ni menos todavía, en cuanto a la variación textual de las composiciones editadas ya que en ningún momento —en línea, en esto, con su predecesor- se puede apreciar un intento de crítica de las variantes. En cambio,

29 Para los criterios de formalización de los aparatos ver Reyes Cano (ed.), "Las Rimas de Juan de la Cueva”, pp. XX-XXI, nota 4. Hay que precisar que es el propio estudioso quien lamenta la escasa fiabilidad textual de estas ediciones, de las que también detecta cada vez el antígrafo entre los 3 testimonios básicos.

30 En realidad, Reyes Cano introduce en su edición una breve nota de la que se desprende que él tenía noticia de algunos otros testimonios manuscritos más, que, sin embargo, no detalla. Aun así, por razones poco comprensibles, a pesar de haber incluido todas las ediciones detectadas, decidió no considerarlos, debido a su estatuto de "copias posteriores no autógrafas". Cfr. Reyes Cano (ed.), "Las Rimas de Juan de la Cueva”, p. XXV. Acerca de la importancia de considerar esta tipología accesoria de fuentes poéticas incluso en los casos en los que se trabaje con autógrafos y otros materiales de autor, nos limitamos a mencionar las páginas modélicas de Alberto Blecua, "Algunas notas curiosas acerca de la transmisión poética española en el siglo XVI", en sus Estudios de crítica textual, Madrid, Gredos, 2012, pp. 233-268 (pp. 263 y ss.).

31 Según el corte del ensayo, edita ora la redacción de las Flores —en "Cueva en Flores de Baria Poesía"—, ora la de las Rimas —en el apéndice del estudio "Juan de la Cueva y Nueva España”, pp. 101-133-. 
es apreciable su esfuerzo de lectura del autógrafo, que le permitió restituir en sus aparatos incluso las lecciones descartadas por el autor y ocultas bajo banderillas, de las que había prescindido totalmente Reyes Cano.

Así las cosas, una nueva edición de estas rimas, concebida según los patrones de la filología de autor ${ }^{32}$, pasa ante todo por una reconsideración del corpus poético, ya que tiene que poner, al lado de los testimonios fundamentales, otros manuscritos e impresos que, al ser vinculados con la persona y con los contextos culturales y literarios del autor, tienen que ser interrogados en cuanto a su estatuto y papel específico, en primer lugar, para la fijación del texto de cada composición en sus múltiples redacciones y, luego, en el estudio de sus variantes y en el consiguiente análisis hermenéutico. Con respecto a los testimonios ya conocidos, considerando sus ya destacadas peculiaridades genéticas y materiales, habrá que volver a interpelarlos detenidamente, a través de una perspectiva filológica más consciente, con el fin de aclarar finalmente la relación entre los testimonios y sus respectivas redacciones y variantes. En concreto, al enfocar este estudio desde la óptica multifocal que requiere el hecho de tener que manejar una tradición mixta, formada además por autógrafos y apógrafos —o sea, por testimonios con niveles de fiabilidad textual diferentes_- habrá que fijarse ante todo en la materialidad de los distintos fascículos de $\mathrm{C} 1$, para intentar relacionarlos, determinar su procedencia y tiempos de composición. Además, y siempre con relación a este testimonio autógrafo, la información relevante sobre los diferentes momentos de copia, el replanteamiento proyectual, la revisión textual y estructural, y el proceso de lima podrá salir de un examen de las variaciones en el ductus y en la grafía de Cueva, así como de los cambios frecuentes en el color y espesor de la tinta, etc. En cuanto al testimonio impreso, habrá que averiguar si existen diferencias entre los distintos ejemplares con que contamos, vista la consabida relevancia de una presunta presencia del autor en el taller de un impresor amigo — como lo fue Pescioni para Cueva-; por consiguiente, será necesario indagar sobre su posible participación en la corrección de pruebas o, al contrario, sobre su desinterés durante el proceso de impresión. Finalmente, no se podrá prescindir de

32 Dentro de la esfera conspicua de modelos y ejemplos proporcionados sobre todo por la escuela filológica italiana, nuestra referencia primaria está representada por Dante Isella, Le carte mescolate vecchie e nuove, Torino, Einaudi, 2009. 
los testimonios "menores" detectados, ya que tanto su exigüidad como su contextualización en buena parte hispalense problematizan el discurso sobre su nivel de fiabilidad y, por consiguiente, sobre la fluidez textual de las composiciones interesadas.

Una vez que se haya fijado el texto de todos los poemas y se haya reconstruido de manera crítica la linealidad de su proceso redaccional y corrector — cuando lo haya, por supuesto_-, se podrá focalizar la atención en la varia lectio así restituida y realizar un estudio detenido de las variantes y variaciones de los poemas, con el fin de reconstruir los rasgos esenciales de la expresión y evolución poética del autor.

Desde esta perspectiva, conviene detenerse un tanto en un aspecto que en los estudios anteriores ha pasado casi desapercibido, es decir, la necesidad de una visión de conjunto de la colección de las Rimas en su estatuto de libro de poemas, fruto de una precisa intentio autori ${ }^{33}$. A ese respecto, no solo es esencial analizar las preferencias temático-formales y dispositivas del autor en un momento histórico de paulatina evolución de formas y modelos en los florilegios poéticos, sino también, desde la perspectiva exclusivamente diacrónica del propio Cueva, dicho poemario exige ser careado con las Obras tanto filológica como críticamente ${ }^{34}$. Efectivamen-

33 De hecho, aunque, como veremos enseguida, lo fuese explícitamente en la perspectiva personal del propio autor, Reyes Cano no restituye de manera alguna la dispositio o el plan de las Rimas en cuanto cancionero; en cambio, en su edición, la ordenación de las composiciones sigue un criterio temático y, secundariamente, cronológico. Cfr. Reyes Cano (ed.), "Las Rimas de Juan de la Cueva”, p. XXXV. Por otra parte, insiste sobre la relevancia de una perspectiva de estudio basada en el enfoque en el libro de poemas del Siglo de Oro como "objeto literario" y "sintagma de relaciones poéticas" - mencionando, además, al propio Cueva entre sus ejemplos-, Valentín Núñez Rivera, "El libro de poemas (1543-1648): tradiciones dispositivas y movilidad textual", en Cancioneros del Siglo de Oro. Forma y formas, ed. Andrea Baldissera, Como/Pavia, Ibis, 2019, pp. 79-102 (pp. 80-81 y 93-96). A este trabajo remitimos también para una bibliografía razonada sobre ese corte metodológico bastante reciente.

34 Que las Obras y, en particular, su primera sección de composiciones breves, constituyan un cancionero, es algo fuera de discusión entre los críticos, aunque personalmente hemos encontrado un solo estudio en el que dicho aspecto se sitúe en el centro de la atención, en concreto: Francisco Javier Burguillo López, "El diseño editorial de las Obras (1582) de Juan de la Cueva", en Cánones críticos en la poesía de los Siglos de Oro, ed. Pedro Ruiz Pérez, Vigo, Academia del Hispanismo, 2008, 
te, si por un lado ya se ha mencionado la recuperación —sometida a revisión- de muchos de los textos preliminares de la antología de 1582 en el paratexto de la recopilación autógrafa, por el otro merece la pena mencionar aquí una declaración muy elocuente del propio Cueva, hecha con ocasión de uno de los primeros sonetos de las Rimas. En concreto, al principio de la colección, tras reproducir los tres mismos sonetos iniciales de las $\mathrm{Obras}^{35}$, el autor introduce un cuarto soneto, Vuestra gloria canté y el ardor mio, a través del cual desvela claramente la operación que está en la base de su nueva recopilación:

Habiendo sido murmurado de algunos el argumento de este libro, porque era solamente amoroso en la primera impresión, se hizo este soneto en que se pide licencia a la señora celebrada en él para juntar diferentes composiciones a diferentes sujetos. Soneto 4. (C1, f. $18 \mathrm{r})^{36}$

De este epígrafe se desprende perfectamente el asunto y el papel del soneto cuarto: el poeta le pide permiso a su dama, que había sido el objeto exclusivo de ese mismo libro en su "primera impresión", para que, al volver sobre él y reformularlo, pueda ahora variar un tanto la perspectiva unifocal allá expresada. Pues bien, la interconexión entre Obras y Rimas, en las intenciones de su autor, no podía ser más explícita ${ }^{37}$. En concreto, precisamente cuando su cancionero amoroso manifiesta la intención de abrirse a nuevos asuntos y temas, bajo el principio de una mayor variatio, Cueva anuncia la conexión genética que el nuevo proyecto mantiene con el primero, convirtiéndolo, de hecho, en el resultado final de una evolución lineal a partir de aquel. Así las cosas, el examen de las variantes tendrá que extenderse necesariamente a las macrovariantes estructurales

pp. 159-169. El estudioso también alude, aunque solo de pasada, a la necesidad de cotejar las intenciones autoriales de las dos recopilaciones (ibidem, pp. 163-164).

35 Para ser más precisos, se invierte en el orden entre el segundo soneto y el tercero.

36 La cursiva es mía.

37 Véanse al respecto las breves pero significativas observaciones de Pedro Ruiz Pérez, “"El riesgo que corren los que comunican sus escritos con el vulgo': las encrucijadas de Juan de la Cueva”, en Cultura oral, visual, y escrita en la España de los Siglos de Oro, eds. José María Díez Borque, Inmaculada Osuna y Eva Llergo, Madrid, Visor, 2010, pp. 515-535 (p. 519). 
entre la "primera impresión" y la segunda, aunque esta haya permanecido atascada en un estadio potencial.

$\mathrm{Ni}$, en realidad, quedan fuera de esta perspectiva de análisis las composiciones juveniles de las Flores, ya que casi todas, como dijimos, entran — revisadas en mayor o menor grado- en las Rimas. Es más, algunas de las variaciones entre $\mathrm{F}$ y $\mathrm{C} 1$ revelan precisamente la sumisión de estos textos a un diseño unitario muy fuerte, de sentido precisamente amoroso. Por sus significativas variantes, es ejemplificador de esta operación de resemantización el soneto Han visto los que viven en la tierra, soneto con dos testimonios: F (pp. 183-184) y C1 (f. 33v). Debido a la fase aún precoz del estudio, se considera aquí más oportuno presentar las dos redacciones en edición sinóptica ${ }^{38}$ :

F

¿Han visto, los que viven en la tierra, el caso extrańo donde vivo y muero?: que huyo del placer y el pesar quiero; que aborrezco la paz y amo la guerra; que sigo a quien me huye y me destierra; 5 tengo esperanza en lo que desespero; en lo más imposible, en eso espero y en eso mi memoria el bien encierra; amo mi duro mal, huyo el contento; fatígame el descanso y su memoria; sigo tras el dolor que me persigue; con lo que me aborrece me contento; llamo a la muerte vida, al amor gloria. Mira en qué pone Amor a quien le sigue.
$\mathrm{C} 1$

¿Han visto, los que viven en la tierra, entre cuántos contrarios vivo y muero?: que huyo del placer y el pesar quiero; que m'ofende la $\mathbf{P A Z}$ y amo la guerra; 5 que sigo a quien me huye y me destierra y espero en lo que más me desespero y, en lo qu'es imposible, el premio espero y voy por donde el paso se me cierra; amo mi duro mal, huyo el contento;

10 fatígame el descanso y su memoria; sigo tras el dolor que me persigue; con lo que m'aborrece me contento; llamo a la muerte vida, al amor gloria; porque tal tiene Amor al que le sigue.

El soneto desarrolla el topos petrarquista de oposición de contrarios que, en la redacción de C1, Cueva decide tematizar (v. 2). Entre las muchas variantes que afloran de un cotejo entre los dos textos, particularmente relevante para nuestro objetivo es la lección del verso 4: "aborrezco la paz"

38 Para los criterios de edición de los textos cfr. supra, nota 3. En cursiva se señalan, aquí y en las citas futuras de F, las lecciones ilegibles en el manuscrito original, subsanadas a través de la copia tardía de Paz y Melia (MN 7892), sobre la que se tendrá ocasión de volver en las próximas páginas; igualmente, en negrita van las variantes entre los testimonios considerados. 
en F; “m’ofende la PAZ” según C1. Ante todo, dada la condición de apógrafo de F, resulta problemático estimar la variación gráfica "paz”/“PAZ”. Por tanto, mejor centrarnos primeramente en la transformación del verbo: “aborrezco"/“m’ofende”. A primera vista, dicha conversión podría explicarse como un intento de variatio, perseguido en $\mathrm{C} 1$, con respecto a la lección del verso 12 ("con lo que me aborrece"), que queda inalterada en las dos redacciones. Sin embargo, a este respecto, conviene notar que no se advierten cambios análogos con relación a otros elementos textuales reiterados (véanse el caso de "huyo", vv. 3 y 9 de ambas redacciones, y de "amo", vv. 4 y 9 tanto en F como en C1). En otras palabras, es la falta de uniformidad variantística del poema con relación a este aspecto la que parece desautorizar la suposición inicial de una intentio auctoris de variación retórico-estilística. Así las cosas, resulta oportuno integrar en el análisis la transformación gráfica del segundo elemento del sintagma del que partimos y pensar en advertir en el uso de la mayúscula por parte de Cueva una funcionalidad específica. De hecho, el juego polisémico y, a veces, incluso acróstico basado en el nombre de la mujer amada es un rasgo estilístico petrarquista muy apreciado por nuestro autor: tanto en el impreso como en el autógrafo, el nombre de doña Felipa de la Paz — sea este el nombre verdadero de la dama, sea su senhal-domina en su poesía amorosa, registrando múltiples recurrencias, totales o bien parciales, en ambos casos oportunamente señaladas gráficamente a través del uso de la mayúscula ${ }^{39}$. En cambio, nunca se le cita a esa mujer en la selección de poemas amorosos incluidos en las Flores. Dos son las conclusiones que se pueden sacar de todo esto. Por un lado, es verosímil suponer, a pesar de la naturaleza de apógrafo de F, que la variación de "paz" a "PAZ" sea una variante de autor, relacionada, quizás, con el episodio biográfico del enamoramiento del poeta y consiguiente conversión de la amada en su musa lírica, episodio que habría que colocar verosímilmente después de la estancia mexicana de nuestro autor y que hizo posible que madurase una lectura polisémica del vocablo "paz", no contemplada originariamente. Siguiendo este razonamiento, para poder efectivamente aprovechar dicha polisemia y hacer posible el nivel segundo de lectura, Cueva tuvo que cambiar el verbo "aborrezco" en "m’ofende”. Además —y es la

39 Sobre el asunto en las Obras véase Burguillo López, "El diseño editorial de las Obras (1582)", pp. 161-164. 
segunda conclusión-, esta interpretación no contrasta con la ausencia del soneto que nos ocupa en $\mathrm{O}$, ya que Cueva pudo haber descartado la composición a la hora de seleccionar un florilegio antológico en nombre de la mujer amada, al no estar efectivamente pensada para ella, mientras que, en un momento sucesivo, al recoger el conjunto de su producción poética, pudo pensar en esta solución para no dejar fuera este soneto y, al mismo tiempo, lograr proporcionar una, por así decirlo, coherencia de inspiración poética en toda su poesía amorosa de marco petrarquista. Dejando para una ocasión futura un comentario más detenido de esta composición, baste por ahora haber destacado la necesidad de perfilar unas instancias autoriales unitarias a las que Cueva, en varios momentos de creación y refundición poética, sometió sus composiciones y recopilaciones.

Por último, esta clave interpretativa, por así decirlo, interna, de las modulaciones del discurso lírico de nuestro poeta en su diacrónica movilidad textual habrá que complementarse con un acertado análisis comparativo de su obra dentro del contexto cultural y literario de las academias y cenáculos sevillanos de esos años al que venimos refiriéndonos desde el principio de estas páginas. Habrá, por tanto, que tener en cuenta tanto la producción de los poetas contertulios suyos como las reflexiones y teorizaciones retóricas y poéticas coevas ${ }^{40}$. Además, considerado el connubio ideal entre teoría y praxis poética perseguido por Cueva, replanteamientos y nuevas soluciones tendrán que ser examinados e interpretados, en particular, a la luz de la aportación teórica del propio autor, desperdigada en las muchas consideraciones metapoéticas incluidas en sus composicio-

40 Además de definir su voz singular dentro del gran universo petrarquista, tendrán que ser analizadas, por ejemplo, su vertiente graciosa dentro del contexto de la poesía sevillana de la sal y su "poética familiar", que tanto le costó en términos de fricciones y enemistades con el círculo herreriano, según las pautas indicadas por Valentín Núñez Rivera, respectivamente en "Otra poesía sevillana del Siglo de Oro. Entre sales y graciosidad", en Literatura y territorio. Hacia una geografia de la creación literaria en los Siglos de Oro, ed. Andrés Sánchez Robayna, Las Palmas de Gran Canaria, Academia Canaria de la Historia, 2010, pp. 513-537 (pp. 521-524); y en "Hacia un doble paradigma. Lírica ornada y poética familiar", en La "Idea" de la Poesía Sevillana en el Siglo de Oro, ed. Begoña López Bueno, Sevilla, Universidad de Sevilla, 2012, pp. 197-254, de las cuales están dedicadas a Cueva y a sus epístolas sobre todo las pp. 239-252. 
nes de varia naturaleza — sobre todo, sus epístolas - y luego recogida en su Exemplar poético ${ }^{41}$.

4. De algunas Flores a las Rimas: un proceso de escritura y ReViSIÓN A TRAVÉS DE TRES DÉCADAS

Vamos a proporcionar ahora un pequeño ejemplo de la investigación in fieri de edición y estudio del corpus de rimas presentado hasta ahora. En concreto, el botón de muestra elegido consta de los 13 poemas atestiguados por los 3 testimonios fundamentales: Flores, Obras y Rimas. Efectivamente, este pequeño grupo de composiciones, todos sonetos con la excepción de una elegía (Robó mi alma un corazón altivo), debido a su significativa extensión temporal representa un muestrario privilegiado para rastrear algunas peculiaridades del proceso de escritura y revisión autorial de Cueva a lo largo de una experiencia poética de más de treinta años. Al mismo tiempo, permite mostrar, a través de ejemplos concretos, los rasgos más problemáticos de tres testimonios tan diferentes como lo son un florilegio manuscrito misceláneo, un libro impreso estando el autor en vida —y, además, atestiguado por muchos ejemplares — y, por último, un autógrafo facticio con diferentes estratos de correcciones y cambios. Por otra parte, hay que señalar que, de estos sonetos, 4 están atestiguados también por otros testimonios, que enriquecen su tradición textual y, por consiguiente, la varia lectio de los correspondientes poemas. Damos a continuación el listado de los poemas considerados:

Íncipit

Amor, de invidia de mi buena suerte

Cantando Orpheo con dorada lira
Testimonios $^{42}$

F, p. 129; O, f. 40v; C1, f. 187v.

F, p. 66; O, f. 89v; C1, f. 366r.

41 La bibliografía sobre el Exemplar es copiosa. Entre los estudios más recientes, mencionamos, por la amplitud de su enfoque, el de Juan José Pastor Comín, "El Exemplar poético de Juan de la Cueva: teoría dramática de un sevillano que pasó sus últimos años en la ciudad de Cuenca”, Archivo Conquense, 2 (1999), pp. 177-210.

42 De momento, pasando por alto el asunto de la cronología entre las redacciones de los textos, el listado de los testimonios se ha ordenado según la cronología de estos últimos. 
Cuando ardió en mí el juvenil brío

Cuando ausente me hallo de mi gloria

Cuando en mi alma represento y miro

Cubrió una oscura noche el día sereno

Dulces regalos de la pena mía

Ira tengo de mí, porque a despecho

Llévame mi deseo a aquella parte

Lleva de gente en gente Amor mi canto

No está en partir mudarse el amor mío

Robó mi alma un corazón altivo

Tantas mudanzas veo en el bien mío
F, p. 158; O, f. 90rv; C1, f. 366r.

F, p. 398; O, f. 70rv; C1, f. 291r.

F, pp. 298-299; O, f. 27v; C1, ff. 68v-69r; MN 3708, f. $65 \mathrm{v}$.

F, p. 68; O, f. 48v; C1, f. 220v.

F, pp. 189-190; O, ff. 74v-75r; C1, f. 324v.

F, p. 262; O, ff. 43v-44r; C1, f. 197r; MN 3708, ff. $62 \mathrm{v}$ y $60 \mathrm{r}^{43}$.

F, p. 298; O, f. 14v; C1, f. 24rv; MN 3708, f. $71 \mathrm{v}$.

F, pp. 107-108; O, f. 90v; C1, f. 366v.

F, p. 397; O, f. 74r; C1, f. 312r.

F, pp. 85-87; O, ff. 21r-22r; C1, ff. 23r-24r;

NYHS B-2369-91, p. 185.

F, p. 362; O, ff. 70v-71r; C1, f. 291v.

Conscientes de lo exiguo del corpus, al par de lo hecho en el apartado anterior, tampoco en este caso se ofrecerá una edición crítica ni se construirán aparatos de variantes; en cambio, sí que se presentarán los resultados de un primer cotejo entre las composiciones consideradas y las observaciones y los comentarios que han derivado de su análisis. Queda por supuesto el carácter parcial y provisional de los resultados, que tendrán que ser investigados y profundizados a la luz de un examen completo del conjunto de las rimas. Mientras tanto, el primer aspecto que el estudio de estas 13 composiciones, en sus diferentes testimonios, ha puesto de manifiesto es una tendencia muy fuerte a la reelaboración por parte de Cueva, ya que se han podido detectar solo dos casos - Cuando ausente me hallo de mi gloria y Lleva de gente en gente Amor mi canto- en los que el texto se mantiene integralmente idéntico en todos sus testimonios; en cambio, en los demás, el autor interviene por lo menos una vez para introducir cambios, aunque frecuentemente mínimos. A pesar de esto, solo

43 La aparente incoherencia de la foliación depende del hecho de que el texto del soneto resulta dividido entre dos folios sueltos, encuadernados desordenadamente en el códice. Cfr. Pablo Jauralde Pou et alii, Catálogo de Manuscritos de la Biblioteca Nacional con poesía en castellano de los siglos XVI y XVII, Madrid, Arco Libros, 19982008, I, pp. 538-555. 
en un caso, el del soneto Ira tengo de mí, porque a despecho, se registran unos cambios realmente notables, que abarcan el sentido y la estructura de toda la composición. En general, así como la entidad de los cambios varía bastante de caso en caso, igual puede decirse con relación a su tipología, ya que, considerando únicamente las variantes sustanciales ${ }^{44}$, estas afectan tanto al léxico — por ejemplo, sinónimos con matices diferentes, pero también cambios más significativos - como a la sintaxis — lo más frecuente son las inversiones entre los sustantivos y sus modificadoreshasta involucrar, aunque solo en unos casos limitados, el sentido global de la frase. De momento, no se ha detectado en los procesos de revisión una conducta unitaria y lineal de trabajo, ya que en algunos casos el texto cambia solo una vez mientras que, en otros, considerando en particular los testimonios básicos, los tres dan fe de otras tantas redacciones. Para ser más precisos, a menudo el texto en su conjunto sigue cambiando al pasar de una redacción a la otra; sin embargo, es bastante raro que una lección dada registre más de un cambio. Tampoco es posible establecer, en términos generales, una mayor o menor proximidad o distancia textual entre las tres redacciones principales al considerarlas de dos en dos, ya que lo que vale para una composición no hay que darlo por descontado para todas las demás. Así, para mencionar solo dos ejemplos opuestos, el texto de C1 de la elegía Robó mi alma un corazón altivo difiere mucho del de $\mathrm{F}$ y $\mathrm{O}$ que, en cambio, resultan más próximos entre sí; en cambio, las variantes más significativas del soneto Ira tengo de mí, porque a despecho se registran en $\mathrm{F}$ mientras que el texto de $\mathrm{O}$ y el de $\mathrm{C} 1$ presentan solo una variante entre ellos.

Así las cosas, hay un aspecto que es necesario enfocar oportunamente. La relación cronológica entre las versiones de $\mathrm{F}, \mathrm{O}$ y $\mathrm{C} 1$ presenta varios problemas ya que, por un lado, tenemos las fechas de los tres testimonios -respectivamente 1577,1582 y 1604 - pero, por el otro, ya hemos mencionado la declaración de Cueva sobre la naturaleza facticia del códice $\mathrm{C}$, que resulta constituido por materiales preexistentes - aunque no siempre tenemos datos extratextuales que nos ayuden a fecharlos con más

44 La naturaleza de los testimonios $\mathrm{F}$ y $\mathrm{O}$, respectivamente apógrafo e impreso, llevan a prescindir de las variantes gráficas, ya que su ortografía ha de interpretarse verosímilmente como el resultado conjunto de los usos gráficos de diferentes agentes intermediarios aparte del autor (copistas, compositores, correctores, etc.). 
precisión- A estas alturas de la investigación, basándonos sobre todo en el pequeño corpus elegido, presentamos algunas consideraciones preliminares. En primer lugar, hay que destacar que los testimonios que han llegado hasta nosotros no son borradores, ni resultan estar todos directamente relacionados entre ellos. De hecho, el único que nos brinda un texto in fieri, o más precisamente un texto base con algunas correcciones y cambios, no parece que haya sido concebido como borrador, sino más bien como una copia en limpio — por lo visto, tenía que ser verosímilmente un original para la imprenta-, aunque el autor aprovechó algunos materiales preexistentes que se encontraban en bastante buen estado en cuanto a claridad del texto. Sin embargo, el punto de partida de C1 consiste en una redacción de los textos poéticos que, en muchos casos, ya no coincide con la de ningún otro testimonio. A este propósito, si no es imposible suponer que Cueva retocase mínimamente el texto al copiarlo de algún borrador en una copia en limpio, parece menos probable que introdujese durante este proceso, es decir, de una forma tan dispersa y aleatoria, cambios más significativos. Puede ser útil al respecto considerar la varia lectio del verso 8 del soneto Amor, de invidia de mi buena suerte, cuyo texto en $\mathrm{C} 1$ resulta diferente de $\mathrm{F}$ y $\mathrm{O}$ ya en su lección base:

\section{F: lo que a entrambos en llanto nos convierte \\ $O$ : lo que a en $[s i c]$ entrambos en llanto nos convierte C1: lo qu'en llanto y en gloria nos convierte}

Por el contrario, de particular interés son las composiciones que en $\mathrm{C} 1$ presentan correcciones por banderillas en correspondencia exacta con todas las lecciones de este testimonio que difieren de la redacción de O. La elegía Robó mi alma un corazón altivo constituye el ejemplo más significativo, sobre todo por la regularidad con la que dicho fenómeno se manifiesta en una composición mucho más larga que un soneto. Efectivamente, en este caso, tan precisa coincidencia sugiere no solo una proximidad entre las dos redacciones de $\mathrm{O}$ y del texto base de $\mathrm{C} 1$, sino incluso una derivación, por así decirlo, directa de la redacción final de C1 de la de O. Aun así, es preciso investigar el asunto con minuciosidad y recurriendo a todas las herramientas codicológicas disponibles antes de formular conclusiones generalizadas, debido a que, por un lado, como hemos visto antes, esta coincidencia textual no vale para todos los textos de C1 que 
cambian con respecto a $\mathrm{O} y$, por el otro, hay también casos de textos en los que se encuentran lecciones finales de $\mathrm{C} 1$ transcritas sobre banderillas que coinciden con las lecciones de $\mathrm{O}$.

Por si fuera poco — y llegamos a la última observación- este primer cotejo ha permitido aislar por lo menos dos casos en los que la versión de C1 no resulta ser legible como resultado de la evolución de un hipotético doble pasaje textual, lineal y directo, de $\mathrm{F}$ a $\mathrm{O}$ y de $\mathrm{O}$ a $\mathrm{C} 1$, que la cronología de los testimonios puede llevar a suponer como el más probable. Para ser más concretos, si observamos las variantes, notamos que la lección del autógrafo parece tener en cuenta ahora la versión de $\mathrm{F}$, ahora la de O. Así, por ejemplo, en el soneto Ira tengo de mí, porque a despecho, en el verso 4 se lee:

\section{F: como en todas las cosas siempre he hecho \\ $\mathrm{O}$ : cual os debe mi fe y siempre lo ha hecho \\ C1: cual os debe mi fe y siempre he hecho}

En este caso $\mathrm{C} 1$ lee igual que $\mathrm{O}$ en la primera parte del verso mientras que coincide con F en la segunda. A su vez, en un par de versos del soneto Cubrió una oscura noche el día sereno (vv. 7-8) se detecta una mayor proximidad textual entre $\mathrm{C} 1$ y $\mathrm{F}$ que respecto a $\mathrm{O}$ :

F: que será inmortal en mi memoria/ en cuanto el mundo de hombres fuere lleno

$O$ : que ha de ser inmortal en mi memoria/ en cuanto el mundo fuere de hombres lleno

C1: que será inmortal en la memoria/ en cuanto el mundo de hombres fuere lleno

A la luz de estos ejemplos, parece verosímil suponer que, entre los papeles que Cueva había recogido para la recopilación de sus Rimas, se encontrase no solo la versión de estos sonetos publicada en las Obras, sino también la que había sido acogida en las Flores. Incluso no sería quizás demasiado atrevido imaginar que, en el momento de la confección de $\mathrm{C} 1$, el autor conservase el manuscrito original que le había servido para la elaboración de las Obras; manuscrito en el que el texto base de algunas composiciones acaso coincidía con el consignado para las Flores, luego revisado y enmen- 
dado - ¿sobre el mismo borrador? - con vista a la publicación de 1582. De esta manera, teniendo delante de sí un texto con varios estratos de escritura, a la hora de intervenir una tercera vez sobre un poema, al lado de los nuevos cambios, Cueva pudo decidir dónde mantener la versión que había dado a la imprenta y dónde, en cambio, era mejor rechazar las variantes introducidas y volver a la primera versión. Considerados los tres testimonios, parece esta la hipótesis reconstructiva, por así decirlo, más económica; aunque, de todas maneras, es evidente que hay que analizar los poemas restantes y buscar otros indicios antes de descartar completamente otras posibilidades que siguen de momento igualmente abiertas. Por ejemplo, nada nos permite descartar, por ahora, incluso la hipótesis de que algunos de los fascículos que componen el autógrafo procedan de materiales preparatorios de $\mathrm{O}$, con un texto sucesivamente abandonado en favor de otras soluciones - las que se leen en $\mathrm{O}-\mathrm{y}$ luego recuperadas y reevaluadas positivamente durante la recolección de textos en $\mathrm{C} 1$. En este caso — no es nada más que una hipótesis — el texto base del autógrafo podría incluso atestiguar un estadio de redacción intermedio entre $\mathrm{F}$ y $\mathrm{O}$ o, ensanchando la mirada —y la suposición — al grupo más amplio de poemas no atestiguados en $\mathrm{F}$ sino exclusivamente en $\mathrm{O}$ y $\mathrm{C} 1$, la de $\mathrm{C} 1$ podría ser una redacción concebida antes de $\mathrm{O} y$, al final, preferida respecto a esta o incluso, en los casos en que el autógrafo lleva correcciones, revisada prescindiendo de la redacción de $\mathrm{O}^{45}$.

Por todo lo visto hasta ahora, las rimas de Juan de la Cueva ofrecen un ejemplo de filología de autor particularmente intricado, al compenetrarse las dos fenomenologías propias de la disciplina, es decir, el original en movimiento y las redacciones múltiples. Sin embargo, como anticipamos, al lado de la complejidad de vinculación entre el autógrafo y los otros dos testimonios fundamentales, otro aspecto que este primer sondeo ha permitido poner de manifiesto consiste en la especificidad de los problemas que plantea cada testimonio. Ya se ha comentado bastante la estratificación genética de $\mathrm{C} 1$; en cuanto a $\mathrm{F}$ y $\mathrm{O}$, ambos son testimonios

45 Con respecto a esta delicada cuestión, será indispensable echar una mirada a algunas obras mayores de nuestro autor y al usus scribendi et corrigendi no siempre lineal con el que trabajó sobre ellas, según dejó reflejado, por ejemplo, en su edición de los dos poemas mitológicos cuevianos el ya citado Cebrián (ed.), "Fábulas mitológicas y épica burlesca", pp. 50-86. 
cuya vinculación con el autor resulta más problemática ya que, en concreto, hay que tomar nota de la mediación de otros agentes —el copista de F, por un lado, y el equipo del taller de imprenta, para el impreso-que pueden interferir en la expresión de la voluntad textual del autor.

En concreto, F presenta los errores de copia propios de los manuscritos apógrafos; sin embargo, la presencia de errores evidentes es la que lleva a dudar, en los casos más conflictivos, es decir, cuando es oportuno hablar más cautelosamente de variantes, sobre la naturaleza de las mismas: variantes de transmisión o de autor. Sirvan como botón de muestra dos variantes mínimas (vv. 10 y 13) del soneto Llévame mi deseo a aquella parte:

F: debilita mi fuerza y enflaquece

$\mathrm{O} / \mathrm{C} 1$ : debilita la fuerza y enflaquece

F: que muerte y vida juntamente ofrece

$\mathrm{O} / \mathrm{C} 1$ : que vida y muerte juntamente ofrece

En realidad, no son estos los únicos problemas que afligen al filólogo a la hora de considerar el texto de la recopilación mexicana. De hecho, el estado de conservación de este manuscrito es lamentablemente muy malo. Aunque no les haya tocado a los poemas de Cueva la peor suerte, la gravedad de su condición es tal que, a veces, igual que ya hicieron las dos editoras del manuscrito, es necesario acudir a la copia realizada por el bibliófilo Paz y Melia en el siglo XIX — es el ms. 7982 de la Biblioteca Nacional de Madrid - para completar sus lagunas. Claramente, aunque esta copia permita evitar algunas cruces desperationis, las lecciones que restituye adquieren forzosamente un nivel aún menos fiable en el discurso sobre variantes de autor. Un caso notable es el del soneto No está en partir mudarse el amor mío, casi completamente ilegible en F. En general, su texto, según la lección de Paz y Melia, no se aleja mucho de las versiones de $\mathrm{O} y \mathrm{C} 1$, pero hay por lo menos dos variantes únicas leídas a través de este testimonio tardío - respectivamente en los vv. 7 y 11 - que convendrá examinar con cuidado ya que, entre las hipótesis posibles, no se puede descartar que formen parte de los no escasísimos errores de mala lectura del bibliófilo:

F: trocar su efecto andando muerte y vida

O/C1: trocar su efecto Amor, muerte dar vida 
F: lo que en sí tiene, sin mudarse della

$\mathrm{O} / \mathrm{C} 1$ : lo que en sí tiene, sin moverse della

Un discurso, en parte, análogo afecta también al testimonio de las Obras. En este caso, hay que enfrentarse con la variabilidad propia de un testimonio impreso en la época de la imprenta manual y hoy atestiguado, como adelantamos, por múltiples ejemplares. En cuanto a este último aspecto, y siempre considerando el muestrario de nuestros 13 poemas, de momento no se han detectado variantes de estado. Con relación a los errores, solo podemos comentar que, frente a fascículos atestiguados en dos fases, con consiguientes correcciones de erratas y errores en algunos de ellos, hay casos de uniformidad en la persistencia de errores evidentes que puede que atestigüen una corrección de pruebas poco cuidadosa o, en cambio, si queremos ser más cautelosos, quizás se deba suponer que simplemente no hemos tenido suerte en la conservación de los ejemplares $^{46}$. De todas formas, la presencia de múltiples ejemplares de la edición facilita la aclaración de la naturaleza de algunas lecciones que, a primera vista, pueden parecer variantes de autor mientras que, tras un cotejo conjunto de los ejemplares y, por supuesto, de las otras dos redacciones de un poema dado en los demás testimonios, desvela que son errores de copia. Un ejemplo para todos puede ser la lección del verso 21 de la elegía Robó mi alma un corazón altivo:

F: vi luego que ver pude a mi enemiga

O: vi luego que ver puede $\left(m_{1566}\right) /$ pude $\left(m_{3267} ; m_{13333} ;\right.$ lon; chi; ox; sal; vie $)^{47}$ mi enemiga

$\mathrm{C} 1$ : vi luego que ver pude mi enemiga

En fin, hasta aquí solo se han considerado las redacciones atestiguadas por los tres testimonios principales. Sin embargo, al llevar la atención a los otros testimonios detectados, entran en juego nuevas variantes. Sirva

46 Quizás esta observación podrá ser algo matizada cuando se tenga la posibilidad de consultar el ejemplar de la Biblioteca del Escorial, que es el único entre los conocidos al que aún no hemos podido acceder.

47 Cfr. supra, nota 20. 
de ejemplo final el soneto Cuando en mi alma represento y miro, copiado también en el testimonio MN 3708; efectivamente, en este manuscrito se registran dos variantes - respectivamente, en los vv. 11 y 14 - que, por pequeñas que sean, destacan por arañar la univocidad de las lecciones correspondientes, en ambos casos, en los tres testimonios de $\mathrm{F}, \mathrm{O}$ y $\mathrm{C} 1$ :

F/O/C1: pues ignoro quién es la que poseo/ dentro en mi alma, donde está tu asiento

MN 3708: pues ignoro quién es la que poseo/ dentro en mi alma $\mathbf{y}$ dónde está su asiento

F/O/C1: no puede ser fingida del deseo,/ ni a otra dar lugar mi pensamiento

MN 3708: no puede ser fingida del deseo,/ ni a otra da lugar mi pensamiento

Como venimos repitiendo desde el principio de este apartado, dichas variantes — así como las consideraciones hasta aquí enunciadas - tendrán que ser interpretadas dentro de un estudio mucho más amplio y profundizado para que los datos filológicos, considerados en un conjunto sistémico, puedan proporcionar una llave de lectura crítica fiable de ese importante componente de la producción literaria de Juan de la Cueva que son sus rimas sueltas. Enmarcada, pues, dentro de sus coordenadas histórico-literarias, la parábola cueviana podrá configurar un válido itinerario para reflexionar sobre la evolución de géneros y modelos, temas y formas, en los fervorosos ańos del humanismo sevillano, representado por autores como Mal Lara y sus discípulos y amigos, y por la experiencia teórico-poética herreriana y de su círculo literario. De hecho, por lo visto, los antagonismos académicos y la relativa marginalidad literaria de nuestro autor no le impidieron jugar su propio papel dentro del vibrante escenario cultural de su tiempo: entre el asentamiento del petrarquismo en el Nuevo Mundo, del que es protagonista, a través del plan de las Flores, al lado de petrarquistas de primera y segunda línea y en diálogo ideal con un autor del calibre de Cetina ${ }^{48}$, hasta el replanteamiento editorial

48 Acerca del asunto véanse, amén de los estudios introductorios a las dos ediciones modernas del florilegio ya citadas, Juan Matas Caballero, "Unas notas sobre el 
tardoquinientista del modelo del cancionero amoroso, en el que participa con su propuesta personal - las Obras - concomitante y alternativa a la de autores como Herrera y Padilla; por último, con el proyecto de un poemario de toda una vida, sus Rimas, no solo se coloca entre una minoría de poetas que, por aquel entonces, llevan adelante el paulatino desarrollo de una atención y un interés personal más marcados, con respecto a unas décadas antes, hacia la transmisión fidedigna —e impresa — de la propia obra completa, sino que también brinda un ejemplo peculiar de la heterogeneidad estructural de los poemarios líricos - ya no son obras las suyas, sino rimas sueltas - al principio del nuevo siglo ${ }^{49}$. En definitiva, adentrarse en la producción lírica de Cueva en su conjunto será una posibilidad más para arrojar nueva luz sobre las múltiples caras de ese escenario poético tan prismático que es el de la ciudad hispalense entre finales del siglo XVI y comienzos del XVII.

petrarquismo en el cancionero Flores de baria poesía", en Luis Barahona de Soto y su época, ed. Antonio Cruz Casado, Lucena, Ayuntamiento de Lucena, 2001, pp. 235-255, quien analiza la asimilación del petrarquismo en las creaciones poéticas de los autores novohispanos incluidos en la antología, suponiendo para ellos el conocimiento previo - y la consiguiente conversión en modelos- de los poemas de Cetina, Herrera, Silvestre y Cueva —entre otros— que luego encontraron acogida en la misma miscelánea.

49 Véase al respecto el ya mencionado estudio de García Aguilar, Poesía y edición en el Siglo de Oro, pp. 215 y ss. 